\title{
Edge Preservation Of Enhanced Fuzzy MEDIAN MEAN FILTER USING DECISION BASED MEDIAN FILTER
}

\author{
Amanpreet Kaur and Ravneet Kaur Sidhu \\ Department of Computer Science, \\ CT Institute of Technology and Research, Jalandhar, India
}

\begin{abstract}
Image noise refers to random variations in the basic characteristics of image like brightness, intensity or color difference. These variations are not present in the image which is captured but may occur due to environmental conditions like sensor temperature or due to circuit of the scanner or other similar issues. Basically noise means unwanted signals in the image. Various filters have been designed for removal of almost all types of noise. It has been seen in most of the cases that as a result of high amount of filtering or repetitive filtering of image for the removal of noise, edges of images mostly get distorted or smeared out. It means that most of the filtering techniques lead to loss of fine edges of the images which needs to be preserved in order to enhance the quality of image. This paper has focused on to improve the enhanced fuzzy median mean filter so that fine edges get preserved in a better way. Experiments have been performed in MATLAB. Comparative analysis have been done on the basis of PSNR, MSE, BER and RMSE and it has shown that border correction applied on images improves the results of enhanced fuzzy median mean filter.
\end{abstract}

\section{KEYWORDS}

Digital Imaging; Noise Smoothing; Edge Distortion; Edge Preservation; Mixed Noise; Enhanced FMM Filter; Border correction

\section{INTRODUCTION}

Digital imaging is the process of creation of digital image such as a scene of a physical object. It includes various activities like sensing of the element by a sensor for acquisition of image, compression of image, storage and hence printing of the image [6]. Noise mostly enters into the image while acquisition process. Imaging is of different types based on electromagnetic radiations or other waves that reflect back from the object while acquisition thereby conveying information about the shape, texture and other features of image [2]. For all classes of images, sensor is responsible for conversion of image information into digital form which are processed by the means of a digital computer so that it is outputted as visible -light image. Medium of visible light allows digital imaging with different types of digital cameras. For instance, X-Rays allow digital $\mathrm{X}$-Ray imaging like radiography, gamma rays allow digital gamma ray imaging like scintigraphy. Almost all image acquiring devices are susceptible to noise which degrades the quality of image [14]. So noise reduction or noise smoothing is the process required for removal of noise from images to improve its quality or in other words to retain the originality of image [7]. Digital image gets affected by various kinds of noise like salt and pepper noise, Gaussian noise, shot noise, speckle noise, gamma noise, Rayleigh noise etc [10]. Any noise reduction algorithm 
International Journal on Soft Computing (IJSC) Vol.7, No. 1, February 2016

attempts to remove noise from image without compromising with or sacrificing the actual details of image. But in most Of The Filtering Techniques It Has Been Seen That Applying Any Filter Risks A Great Loss Of Original Fine Details Of The Image. A Dedicated Filtering Technique Aims At Retaining Originality Of Image As Far As Possible [11]. Various Techniques Exist For Smoothing Of Images Like Use Of Linear Or Non-Linear Filters, Anisotropic Diffusion Etc. Linear Filters Work By Convolving Image With Spatial Mask Which Represents Smoothing Operation [4] [10]. The Purpose Of Convolution Process Is To Bring The Value Of Each And Every Pixel In Close Harmony To Its Neighboring Pixels. Anisotropic Diffusion Evolves Image Under A Partial Differential Equation For Smoothing. Non-Linear Filters Sort Pixels Of Images According To Their Intensities And Later Replace The Noisy Pixels By Best Alternatives Available. Wavelet Transform Technique Can Also Be Used For Both Noise Reduction As Well As Feature Preservation. According To This Technique Noise Is Uniformly Spread Throughout The Coefficients Of Image And The Original Information Of Image Is Concentrated In Few Large Coefficients. Certain Statistical Methods Also Exist For The Removal Of Noise From Images [8] [11]. Applying Almost All Of The Above Mentioned Noise Smoothing Techniques Result In Loss Of Edges Of Images As Repetitive Smoothing Results In Smearing Out Of Images. So Sharp Edges Of Images Need To Be Retained After Removal Of Noise From Them. Edge Preserving Filters Can Be Applied On Digital Images As Post Processing Technique To Further Enhance The Visual As Well As Statistical Quality Of Image [3]. Various Edge Preserving Filters Exist For This Purpose Like Bilateral Filter, Trilateral Filter And Guided Image Filter Etc [1]. This Post Processing Needs To Be Performed On Image Because Even If Denoising Does Not Completely Remove The Boundaries Or Border Of An Image, It Does Distort Sharp Edges To Some Or To Large Extent. Distortion Of Fine Boundaries Of Images Is Not Acceptable Even If Noise Gets Completely Removed From The Image Especially In The Case Of Medical Images [5]. The Outline Of This Paper Is As Follows. Section 2 Describes Enhanced Fuzzy Median Mean Filter And Section 3 Describes A Method To Improve This Enhanced FMM Filter Further. Experiments And Results Are Shown In Section 4. Conclusion Is Given In Section 5.

\section{EnhanCEd FuZzy Median Mean (FMm) FiLter}

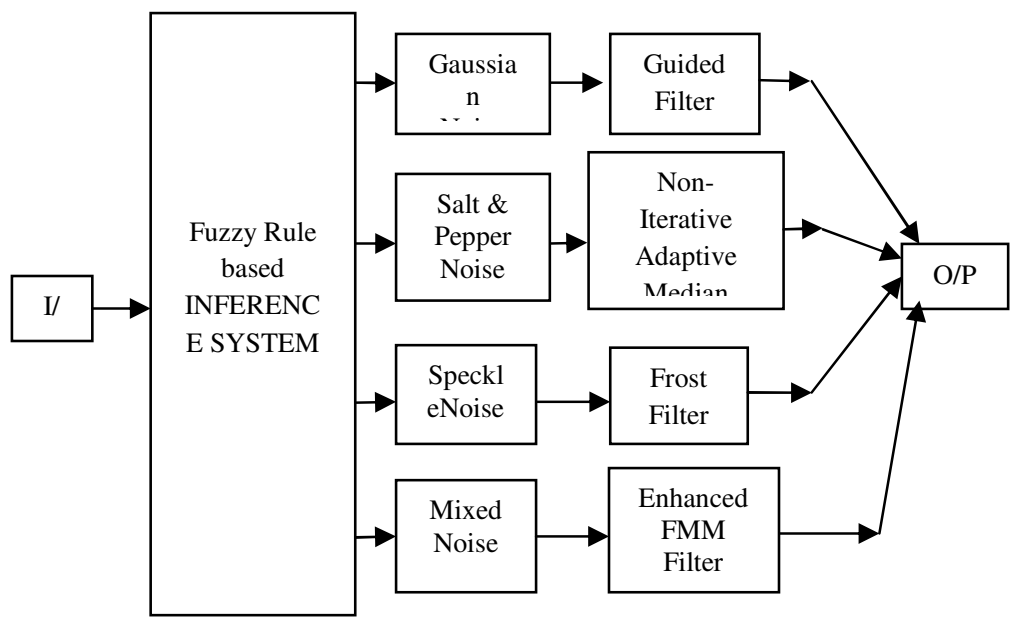

Figure 1. Flowchart describes working of Enhanced FMM Filter 
Fuzzy Inference Systems (FIS) Can Be Used To Fuzzify The Inputs Into Membership Functions. They Operate In Steps. Firstly They Combine The Fuzzified Inputs According To Fuzzy Rules To Establish Rule Strength. Then Consequence Of The Rule Is Found By Combining The Rule Strength And Output Membership Function. Later On The Consequences Are Combined To Get An Output Distribution. At The Last Stage, Output Distribution Is Defuzzified. Fuzzy Logic Have Been Used Successfully To Remove Mixed Type Of Noise Comprising Any Of The Gaussian Noise, Salt And Pepper Noise, Speckle Noise Or combination of any of these three. It was used to enhance the Fuzzy Median Mean filter which could only remove two types noise namely Salt and Pepper noise and Gaussian noise [20]. Newly developed Enhanced Fuzzy Median Mean filter is now capable of removal of three types of noise whether any one of these is present in image or all three are present. It is suitable for their combinations as well. Enhanced FMM makes use of Guided image filter for Gaussian noise, Non-Iterative Adaptive Median filter for Salt \& Pepper noise and Frost filter for Speckle noise respectively [15] [19] [21]. It proved that expert knowledge or data mining based fuzzy logic can help to solve complex problems like removal of mixed noise from images which was not possible earlier. It makes robust decisions [20].

\section{PROPOSED METHODOLOGY}

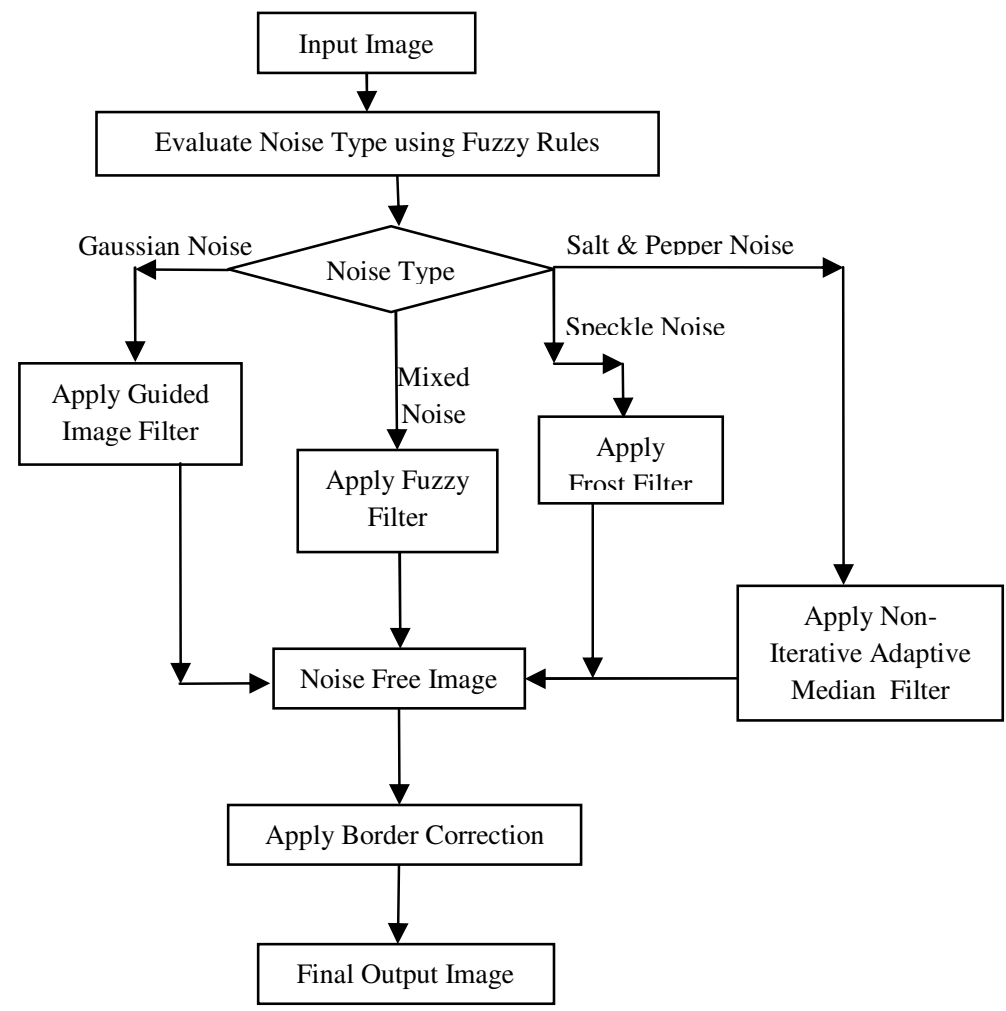

Figure 2. Flowchart describes working of Enhanced FMM Filter 
Although, the application of various filters in Enhanced Fuzzy Median Mean Filter have successfully removed combination of three different types of noises from it but it has led to edge distortion. Since noise reduction or noise smoothing also involves preserving fine details of image including sharp edges so it has been accomplished by applying certain border correction as post processing after noise removal from images [11] [16]. Comparison of two edge preserving filters namely Switching mean median filter and Decision based median filter using morphological operator have been done on the basis of certain quality parameters. It is evident from the comparatative analysis that Decision based median filter preserve edges in a much better way.

\section{RESULTS \& DiSCUSSIONS}

Experiments have been performed for different combinations of noise densities for 15 different images taken from a database but results for two noise densities have been shown for 10 images.

\subsection{Results of Enhanced Fuzzy Median Mean Filter at low noise density}

Experiments have been performed by adding three types of noise into the image, i.e. Gaussian noise at 0 Mean and 0.01 Standard Deviation, Salt \& Pepper noise with $15 \%$ density and Speckle noise with $10 \%$ density i.e. all noises with low density. Results of Enhanced FMM show that it works effectively in the presence of three types of noises i.e. Gaussian, Salt \& Pepper and Speckle all at the same time which is not possible in the case of FMM filter.

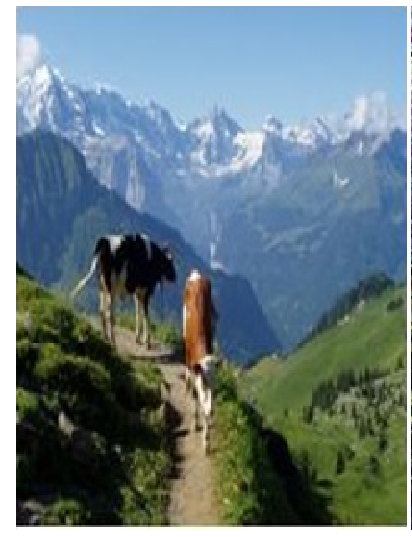

(a)

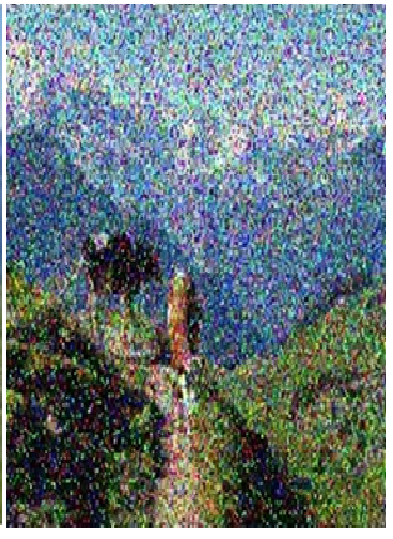

(b)

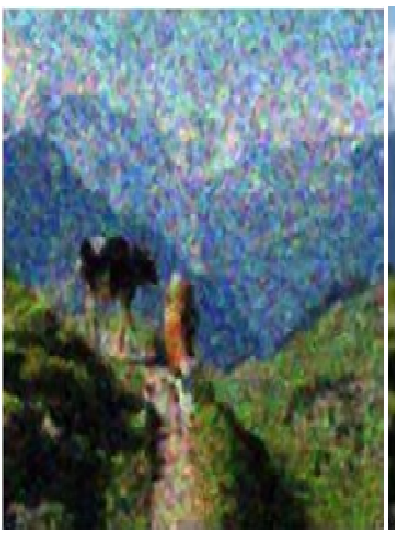

(c)

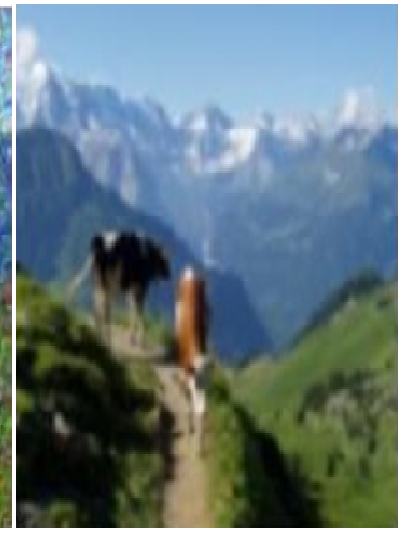

(d)

Figure 3. (a) Input Image (b) Noisy Image (C) FMM Filtered Image (d) Enhanced FMM Filtered Image

\subsection{Results of Enhanced Fuzzy Median Mean Filter at high noise density}

Experiments have been performed by adding three types of noise into the image, i.e. Gaussian noise at 0 Mean and 0.03 Standard Deviation, Salt \& Pepper noise with 75\% density and Speckle noise with $30 \%$ density i.e. all noises with high density. Results of Enhanced FMM show that it works effectively in the presence of three types of noises i.e. Gaussian, Salt \& Pepper and Speckle all at the same time which is not possible in the case of FMM filter. 
International Journal on Soft Computing (IJSC) Vol.7, No. 1, February 2016

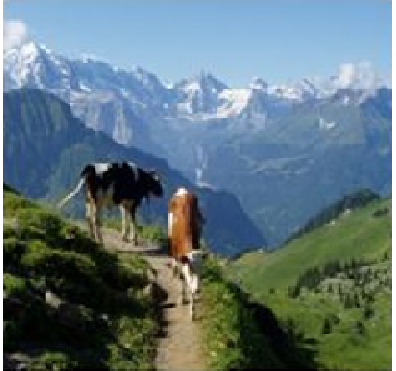

(a)

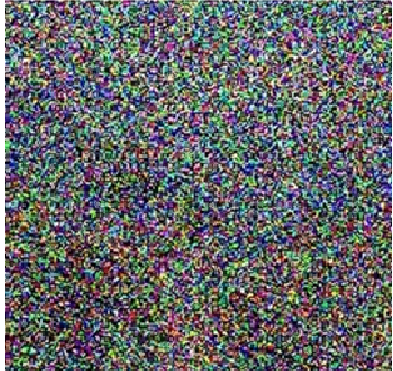

(b)

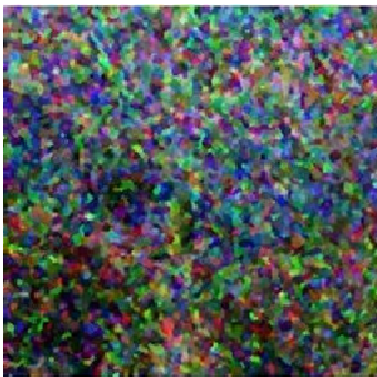

(c)

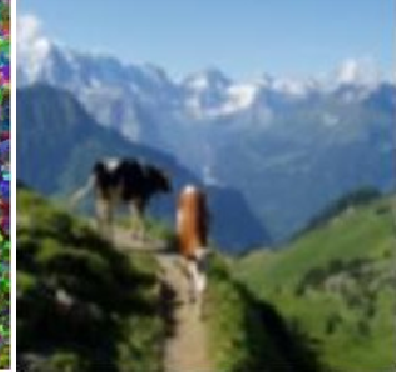

(d)

Figure 4. (a) Input Image (b) Noisy Image (C) FMM Filtered Image (d) Enhanced FMM Filtered Image

\subsection{Results of EFMM with edge preservation at low noise density}

Experiments have been performed by adding three types of noise into the image, i.e. Gaussian noise at 0 Mean and 0.01 Standard Deviation, Salt \& Pepper noise with $15 \%$ density and Speckle noise with $10 \%$ density.

Figure 3 (a) shows the input image that has been chosen from the database. Three types of noise have been added into the image i.e. 15\% Salt \& Pepper noise, Gaussian noise with 0 mean and 0.01 standard deviation and Speckle noise with $10 \%$ noise density which has been shown in (b) part. After that noise is being removed from noisy image by applying Enhanced Fuzzy Median Mean (FMM) Filter as shown in (c). Then the image is denoised by applying edge preserving Decision based median filter using morphological operator i.e. as shown in (d).

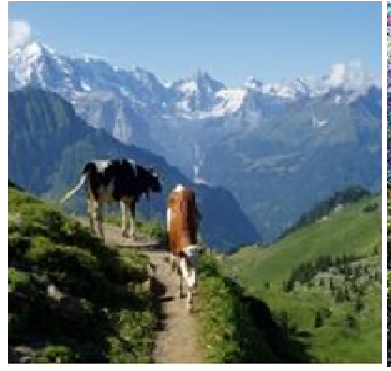

(a)

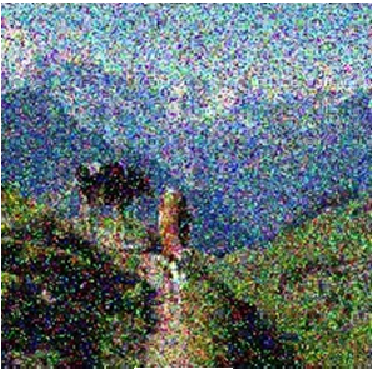

(b)

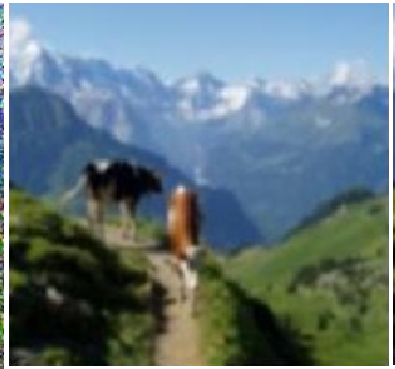

(c)

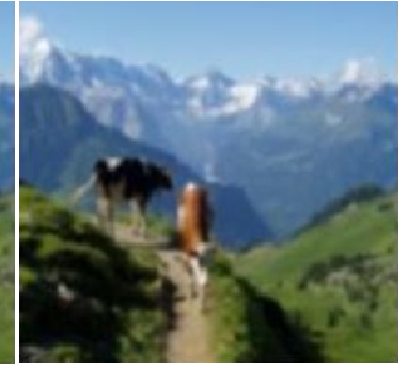

(d)

Figure 5. (a) Input Image (b) Noisy Image (c) Enhanced FMM Filtered Image (d) Enhanced FMM Filtered Image after Edge Preservation

\subsection{Results of EFMM with edge preservation at high noise density}

Experiments have been performed by adding three types of noise into the image, i.e. Gaussian noise at 0 Mean and 0.03 Standard Deviation, Salt \& Pepper noise with 75\% density and Speckle noise with $30 \%$ density.

Figure 4(a) shows the input image that has been chosen from the database. Three types of noise have been added into the image i.e. Salt \& Pepper noise with $75 \%$ noise density, Gaussian noise with 0 mean and 0.03 standard deviation and Speckle noise with $30 \%$ noise density which has been shown in (b) part. After that noise is being removed from noisy image by applying 
Enhanced Fuzzy Median Mean Filter as shown in (c). Then the image is denoised by applying edge preserving Decision based median filter using morphological operator as shown in $(\mathrm{d})$.

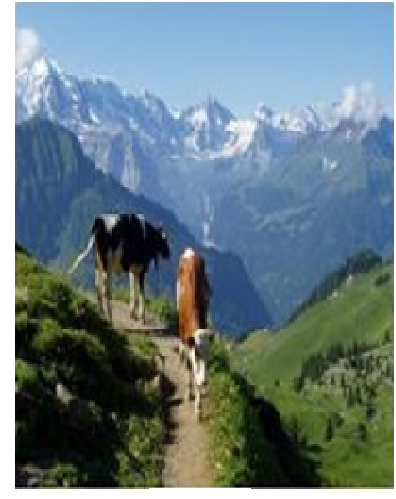

(a)

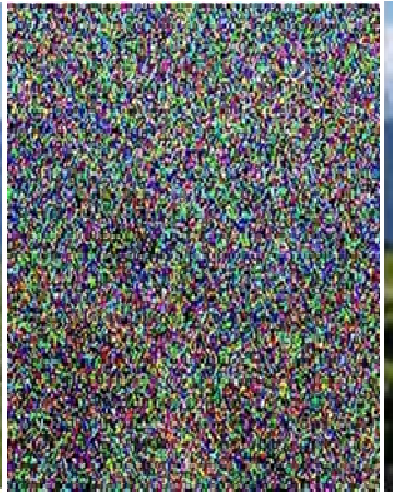

(b)

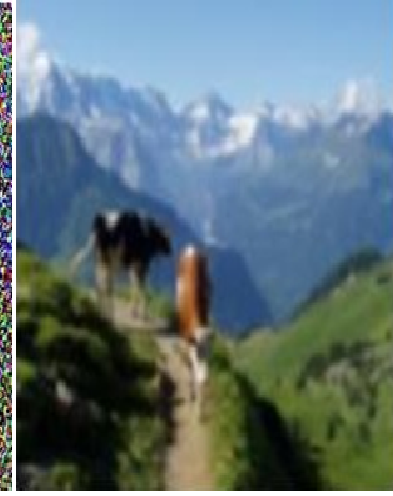

(c)

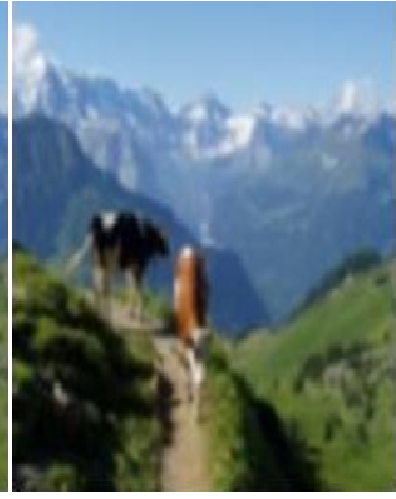

(d)

Figure 6. (a) Input Image (b) Noisy Image (c) Enhanced FMM Filtered Image (d) Enhanced FMM Filtered Image after Edge Preservation

\section{Performance Evaluation}

Comparison of two edge preserving filters namely Switching Mean Median Filter and Decision based Median Filter using morphological operator have been done. Comparative analysis have shown that Decision based median filter preserves edges in a much better way and thus it has been used for the same purpose. Four parameters have been used for this comparative analysis namely MSE, PSNR, BER and RMSE.

Following tables show the comparison of results of Enhanced Fuzzy Median Mean Filter and Decision based median filter using morphological operator after applying border correction on it for $75 \%$ Salt \& Pepper noise density, 30\% Speckle noise density, Gaussian noise at 0 mean and 0.03 standard deviation. Quality parameters have been used to compare the performance of Enhanced Fuzzy Median Mean Filter both before and applying border correction on it.

\subsection{Mean Square Error (MSE)}

Mean Square Error in image processing measures the average of squares of errors. This error denotes the difference between estimator and what is actually estimated. MSE can be measured by the following expression:

$$
M S E=\frac{1}{X Y} \sum_{x=1}^{X} \sum_{y=1}^{Y}\left(f(x, y)-f^{\prime}(x, y)\right)^{2} \quad . . \mathrm{Eq}^{\mathrm{n}}(1)
$$

MSE values for Switching mean median filter and decision based median filter are 129 and 123 respectively which indicates that mean square error is lesser in the case of decision based median filter and hence it has been chosen for border correction.

Table 1 shows the values for Mean Square Error for Noisy Image, Enhanced FMM Filtered Image before and after applying border correction on it respectively for 10 different color images chosen from a database. 
International Journal on Soft Computing (IJSC) Vol.7, No. 1, February 2016

Table 1. MSE Values

\begin{tabular}{|c|c|c|c|}
\hline IMG & $\begin{array}{c}\text { Noisy } \\
\text { Image }\end{array}$ & $\begin{array}{c}\text { Enhanced FMM Filter } \\
\text { before border correction }\end{array}$ & $\begin{array}{c}\text { Enhanced FMM Filter } \\
\text { after border correction }\end{array}$ \\
\hline 1 & 10769 & 86 & 71 \\
\hline 2 & 11120 & 108 & 91 \\
\hline 3 & 10557 & 98 & 80 \\
\hline 4 & 8676 & 143 & 123 \\
\hline 5 & 9657 & 111 & 96 \\
\hline 6 & 9945 & 214 & 900 \\
\hline 7 & 9321 & 109 & 167 \\
\hline 8 & 9117 & 181 & 173 \\
\hline 9 & 8789 & 187 & 73 \\
\hline 10 & 12757 & 90 & \\
\hline
\end{tabular}

\subsection{Peak Signal to Noise Ratio (PSNR)}

Peak Signal to Noise Ratio is used basically to measure the accuracy of reconstruction of lossy compression codecs. Signal here denotes the original data while Noise refers to the error that is introduced during compression of image. For higher quality reconstruction, PSNR value must be high. It can be measured by the following expression:

$$
\begin{gathered}
P S N R=10 \log _{10}\left(M A X_{f}^{2} \mid M S E\right) \\
=20 \log _{10}\left(M A X_{f}\right)-10 \log _{10}(M S E) \quad . . \mathrm{Eq}^{\mathrm{n}}(2)
\end{gathered}
$$

PSNR values for Switching mean median filter and decision based median filter are 26.826 and 27.376 respectively which indicates that signal to noise ratio is higher in the case of decision based median filter which means that quality of signals will be better in case of images in which edges are preserved by using decision based median filter and hence it has been chosen for border correction.

Table 2 shows the values for Mean Square Error for Noisy Image, Enhanced FMM Filtered Image before and after applying border correction on it respectively for 10 different color images chosen from a database.

Table 2. PSNR Values

\begin{tabular}{|c|c|c|c|}
\hline IMG & $\begin{array}{c}\text { Noisy } \\
\text { Image }\end{array}$ & $\begin{array}{c}\text { Enhanced FMM Filter } \\
\text { before border correction }\end{array}$ & $\begin{array}{c}\text { Enhanced FMM Filter } \\
\text { after border correction }\end{array}$ \\
\hline 1 & 7.8090 & 28.785 & 29.987 \\
\hline 2 & 7.669 & 27.796 & 28.978 \\
\hline 3 & 6.9561 & 28.561 & 29.568 \\
\hline 4 & 8.7476 & 26.577 & 27.376 \\
\hline 5 & 8.7751 & 27.661 & 28.563 \\
\hline 6 & 8.1548 & 24.826 & 25.882 \\
\hline 7 & 8.7651 & 27.661 & 28.251 \\
\hline 8 & 8.7521 & 28.161 & 29.244 \\
\hline 9 & 9.6761 & 27.124 & 28.945 \\
\hline 10 & 7.1161 & 27.511 & \\
\hline
\end{tabular}




\subsection{Bit Error Rate (BER)}

Bit Error Rate basically measures the bit errors that occur in digital data traffic for a particular time interval. For this purpose, it calculates the ratio between bit errors and total number of bits that are transmitted for that interval. It can be calculated by the expression:

$$
B E R=100 \times(1-B C R)
$$

where BCR is Balanced Classification Rate

$$
=\frac{N_{\text {error }}}{N_{\text {bits }}} \quad \ldots \mathrm{Eq}^{\mathrm{n}}(3)
$$

BER values for Switching mean median filter and decision based median filter are 0.0322 and 0.0278 respectively which indicates that bit error rate is lesser in the case of decision based median filter which means that during transmission bit errors will be lesser in case of images in which edges are preserved by using decision based median filter hand hence it has been chosen for border correction.

Table 3 shows the values for Mean Square Error for Noisy Image, Enhanced FMM Filtered Image before and after applying border correction on it respectively for 10 different color images chosen from a database.

Table 3. BER Values

\begin{tabular}{|c|c|c|c|}
\hline IMG & $\begin{array}{c}\text { Noisy } \\
\text { Image }\end{array}$ & $\begin{array}{c}\text { Enhanced FMM Filter } \\
\text { before border correction }\end{array}$ & $\begin{array}{c}\text { Enhanced FMM Filter } \\
\text { after border correction }\end{array}$ \\
\hline 1 & 0.1281 & 0.0347 & 0.0269 \\
\hline 2 & 0.1304 & 0.0360 & 0.0225 \\
\hline 3 & 0.1299 & 0.0321 & 0.0223 \\
\hline 4 & 0.1143 & 0.0376 & 0.0278 \\
\hline 5 & 0.1212 & 0.0299 & 0.0196 \\
\hline 6 & 0.1226 & 0.0403 & 0.0302 \\
\hline 7 & 0.1232 & 0.0299 & 0.0194 \\
\hline 8 & 0.1312 & 0.0199 & 0.0078 \\
\hline 9 & 0.1287 & 0.0891 & 0.0601 \\
\hline 10 & 0.1399 & 0.0521 & 0.0276 \\
\hline
\end{tabular}

\subsection{Root Mean Square Error (RMSE)}

It is a measure of difference between values of estimator and values that are actually estimated. It presents standard deviation of mean square error. It can be measured by the expression:

$$
R M S E=\sqrt{M S E} \quad \ldots \mathrm{Eq}^{\mathrm{n}}(4)
$$

RMSE values for Switching mean median filter and decision based median filter are 11.453 and 10.973 respectively which indicates that root mean square error is lesser in the case of decision based median filter and hence it has been chosen for border correction.

Table 4 shows the values for Mean Square Error for Noisy Image, Enhanced FMM Filtered Image before and after applying border correction on it respectively for 10 different color images chosen from a database. 
Table 4. RMSE Values

\begin{tabular}{|c|c|c|c|}
\hline IMG & $\begin{array}{c}\text { Noisy } \\
\text { Image }\end{array}$ & $\begin{array}{c}\text { Enhanced FMM Filter } \\
\text { before border correction }\end{array}$ & $\begin{array}{c}\text { Enhanced FMM Filter } \\
\text { after border correction }\end{array}$ \\
\hline 1 & 103.773 & 9.2736 & 8.2469 \\
\hline 2 & 105.452 & 10.392 & 9.358 \\
\hline 3 & 104.895 & 9.3190 & 8.213 \\
\hline 4 & 93.1452 & 11.958 & 10.973 \\
\hline 5 & 97.8981 & 9.861 & 8.997 \\
\hline 6 & 99.7246 & 14.6287 & 13.234 \\
\hline 7 & 97.8181 & 9.861 & 8.902 \\
\hline 8 & 97.8181 & 9.111 & 8.238 \\
\hline 9 & 98.787 & 9.8712 & 8.7821 \\
\hline 10 & 124.895 & 9.3390 & 8.342 \\
\hline
\end{tabular}

\section{CONCLUSION}

This paper has improved the Enhanced Fuzzy Median Filter used for the removal of mixed noise (Salt and Pepper noise, Gaussian noise and Speckle noise) from digital images. It was clear from the analysis that no doubt Enhanced FMM successfully removes three types of noises from digital images but it distorts edges to some extent which is not acceptable. This is because each and every minute detail of image is very important to define it completely. So this work has focused on to preserve sharp edges of images denoised by EFMM i.e. Enhanced Fuzzy Median Mean Filter. Two edge preserving filters namely Switching mean median filter and Decision based median filter using morphological operator have been applied on images filtered by Enhanced fuzzy median mean filter. Comparative analysis have been done on the basis of four quality parameters namely Mean square error, Peak signal to noise ratio, Bit error rate and Root mean square error which shows that edge preserving Decision based Median Filter has given optimized results. Hence this filter has been chosen for edge preservation of images filtered by EFMM. Filters have been designed in image processing toolbox of MATLAB.

\section{REFERENCES}

[1] R.T. Chin and C. L. Yeh. "Quantitative Evaluation of Some Edge-Preserving Noise-Smoothing Techniques", Elsevier Journal of Graphical Models and Image Processing, Vol. 23, No. 1, pp. 67-91, 1983.

[2] S.E. Reichenbach, S.K. Park and R. Narayanswamy, "Characterizing Digital Image Acquisition Devices", SPIE Digital Library, Vol. 30, No. 2, pp. 170-177, 1991.

[3] Z. N. Li and G. Hu, "On Edge Preservation in Multiresolution Images", Elsevier Journal of Graphical Models and Image Processing, Vol. 54, No. 6, pp. 462-471, 1992.

[4] K. Tang, J. Astola and Y. Neuvo, "Non-Linear Multivariate Image Filtering Techniques", IEEE Transactions on Image Processing, Vol. 4, No. 6, pp. 788-798, 1995.

[5] P. Charbonnier, L. Blanc-Feraud, G. Aubert and M. Barlaud, "Deterministic Edge-Preserving Regularization in Computed Imaging", IEEE Transactions on Image Processing, Vol. 6, No. 2, pp. 298-311, 1997.

[6] G. Sharma and H. Trusell, "Digital Color Imaging", IEEE Transactions on Image Processing, Vol. 6, No. 7, pp. 901-932, 1997. 
International Journal on Soft Computing (IJSC) Vol.7, No. 1, February 2016

[7] G.M. Johnson, M.D. Fairchild and M. Garrett, "The Effect of Opponent Noise on Image Quality", International Society for Optics and Photonics in Electronic Imaging, pp. 82-89, 2005.

[8] V.R.Vijaykumar, P.T.Vanathi, P.Kanagasabapathy, "Fast and Efficient Algorithm to remove Gaussian Noise in Digital Images", IAENG International Journal of Computer Science, no. 1, pp. 300-302, 2010.

[9] E. Lopez-Rubio, "Restoration of images corrupted by Gaussian and uniform impulsive noise", Elsevier International Journal of Pattern Recognition, Vol. 43, No. 5, pp. 1835-1846, 2010.

[10] C. Mythili and V. Kavitha, "Efficient Technique for Color Image Noise Reduction", The Research Bulletin of Jordan, Vol. 1, No. 11, pp. 41-44, 2011.

[11] S.R. Mahakale and N.V. Thakur, "A Comparative Study of Image Filtering on Various Noisy Pixels", International Journal of Image Processing and Vision Sciences, Vol. 1, No. 2, pp. 69-77, 2012.

[12] B. Mohd. Jabarullah, S. Saxena and Dr. C. Nelson Kennedy Babu, "Survey on Noise Removal in Digital Images", IOSR Journal of Computer Engineering (IOSRJCE), Vol. 6, No. 4, pp. 45-51, 2012.

[13] R.R. Varade, M.R. Dhotre and A.B. Pahurkar, "A Survey on Various Median Filtering Techniques for Removal of Impulse Noise from Digital Images", International Journal of Advanced Research in Computer Engineering \& Technology, Vol. 2, No. 2, pp. 606-609, 2013.

[14] R. Verma and J. Ali, "A Comparative Study of Various Types of Image Noise and Efficient Noise Removal Techniques", International Journal of Advanced Research in Computer Science and Software Engineering, Vol. 3, No. 10, pp. 617-622, 2013.

[15] K. He, J. Sun and X. Tang, "Guided Image Filtering", IEEE Transactions On Pattern Analysis And Machine Intelligence, Vol. 35, No. 6, pp. 1397-1409, 2013.

[16] P. Singh and A. Arora, "Analytical Analysis of Image Filtering Techniques", International Journal of Engineering and Innovative Technology (IJEIT), Vol. 3, No. 4, pp. 29-32, 2013.

[17] P. Agrawal and J. Singh Verma, "A Survey of Linear and Non-Linear Filters for Noise Reduction", International Journal of Advance Research in Computer Science and Management Studies, Vol. 1, No. 3, pp. 18-25, 2013.

[18] E. Simrat and E. Anil Sagar, "Empirical Study of Various Speckle Noise Removal Methods", International Journal of Emerging Technologies in Computational and Applied Sciences (IJETCAS), pp. 513-516, 2014.

[19] V. Bhateja, K. Rastogi, A. Verma and C. Malhotra, "A Non-Iterative Adaptive Median Filter for Image Denoising", IEEE International Conference on Signal Processing and Integrated Networks (SPIN), pp. 113-118, 2014.

[20] Y. Wang, G. Wu, G. (Sheng) Chen and T. Chai, "Data mining based noise diagnosis and fuzzy filter design for image processing", Elsevier Journal of Computers and Electrical Engineering, Vol. 40, No. 7, pp. 2038-2049, 2014.

[21] A. Kaur, R. Malhotra and R. Kaur, "Performance evaluation of non-iterative adaptive median filter", IEEE International Advance Computing Conference (IACC), pp. 1117-1121, 2015.

[22] O.D. Fenwa, F.A. Ajala and O.T. Adedeji, "Performance Evaluation of Selected Noise Removal Algorithms in Sickle Cell Images", International Journal of Emerging Trends \& Technology in Computer Science (IJETTCS), Vol. 4, No. 1, pp. 1-5, 2015.

[23] U. Bansal, R. Saini and A. Verma, "A New Methodology for Sp Noise Removal in Digital Image Processing", International Journal in Foundations of Computer Science \& Technology (IJFCST), Vol. 5, No. 1,pp. 67-73, 2015. 